\title{
COMPORTAMIENTO DE COMBINACIONES PORTAINJERTO-CULTIVAR DE PLANTAS JÓVENES DE MANZANOS DE BAJO REQUERIMIENTO DE HORAS DE FRÍO EN SAN LUIS DEL PALMAR (CORRIENTES - ARGENTINA)
}

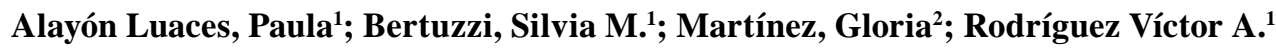 \\ ${ }^{1}$ Cátedra de Fruticultura, ${ }^{2}$ Cátedra de Química General e Inorgánica - \\ Facultad de Ciencias Agrarias (UNNE) Sargento Cabral 2131 (3400) Corrientes. \\ Correo electrónico: p_alayon@agr.unne.edu.ar
}

\begin{abstract}
RESUMEN
Desde el año 2001 se cultivan en San Luis del Palmar (Corrientes) manzanos de bajo requerimiento de horas de frío. Pocos son los antecedentes de este cultivo en el Nordeste Argentino y si bien los hay en Cerro Azul (Misiones), de donde provienen los cultivares y combinaciones, las características agro ecológicas de ambas localidades son sustancialmente diferentes. Con el objetivo de evaluar el comportamiento de combinaciones portainjertocultivar de manzanos de bajos requerimientos de horas de frio en San Luis del Palmar (Corrientes), se realizaron determinaciones vegetativas y productivas del cultivo durante las campañas 2003/2004; 2004/2005 y 2005/2006. Se estudiaron tres cultivares: “Anna”, "Eva” y "Caricia” combinados con tres portainjertos: “EM9”, “MM111” y "Pie Correntino (PC)". De las combinaciones evaluadas la que presentó mayor eficiencia de cultivo fue Anna/EM9 (eficiencia promedio de las tres campañas $279 \mathrm{~g} \mathrm{~cm}^{-2}$ ) y la de menor eficiencia Eva/ MM111 (eficiencia promedio de las tres campañas $117.83 \mathrm{~g} \mathrm{~cm}^{-2}$ ). El cultivo de manzanos de bajos requerimientos de horas de frío es factible en las condiciones agroecológicas de San Luis del Palmar - Corrientes y la combinación más eficiente fue Anna/EM9.
\end{abstract}

PALABRAS CLAVES: manzanos subtropicales, eficiencia de cultivo, NEA Argentino.

\section{SUMMARY}

Since 2001 in San Luis del Palmar Corrientes Argentina low chill apple cultivars has been farmed. There are not many antecedents about this crop in the Argentinean Northeast, the most important reported are from Cerro Azul (Misiones-Argentina) where the apple cultivars are from, but the agro ecological condition are substantially different in Corrientes with respect to Misiones. With the objective to evaluate different combination of rootstocks-cultivars of low chill apple behaviour in Corrientes, vegetative and productive determi- nations has been done during three consecutives farm years 2003/2004; 2004/2005 and 2005/2006. Three cultivars "Anna”, "Eva” and "Caricia” all of them grafted on three rootstocks: "EM9”, "MM111” and "Pie Correntino (PC)" has been evaluated. The low chill apple varieties crop is feasible under the agro ecological conditions in San Luis del Palmar Corrientes and Anna/EM9 showed the best crop efficiency.

KEY WORDS: subtropical apple; crop efficiency, Argentinean Northeast

\section{INTRODUCCION}

El cultivo del manzano (Malus domestica Borkh) es conocido por su alto requerimiento en horas de frío, sin embargo existen grandes diferencias entre cultivares, con oscilaciones de requerimientos que van desde las 200 a las 1400 horas de frío (Samson, 1991). La mayoría de los cultivares antiguos presentan requerimientos de frío más acentuados para la ruptura de la dormición de sus yemas, no obstante en la actualidad y merced al mejoramiento genético, se cuenta con cultivares de bajos requerimientos de horas de frío y con frutos de buena calidad.

La Fruticultura en el Nordeste Argentino (NEA) se limita básicamente a cultivos citrícolas (naranjas, mandarinas y limones) y casi no se realizan plantaciones frutícolas comerciales de importancia de otras especies. Esto genera la necesidad de buscar alternativas frutícolas agronómicamente factibles y económicamente viables que brinden a los productores un cultivo intensivo y rentable para diversificar la producción de frutas de la zona.

En la localidad de San Luis del Palmar (Corrientes) se cultivan manzanas en forma comercial desde el año 2001 con cultivares de los que se denominan de bajo requerimiento de horas de frío. En nuestro país son escasos los antecedentes de investigaciones científico tecnológicas en estos cultivares, los que se destacan son los realizados en el INTA Cerro Azul (Misiones) (Provasi et al, 2000) y en 
Colonia Caroya (Córdoba) (Páez Morón, 2005), sin embargo en dichas zonas las condiciones agroclimáticas difieren sustancialmente de las que se presentan en la zona de Corrientes.

El objetivo de este trabajo fue evaluar el comportamiento de combinaciones portainjerto-cultivar de manzanos de bajos requerimientos de horas de frío en San Luis del Palmar (Corrientes), por medio de determinaciones vegetativas y productivas del cultivo durante las campañas 2003/2004; 2004/2005 y 2005/2006.

\section{MATERIALES Y MÉTODOS Material Vegetal}

Las evaluaciones y determinaciones se realizaron en frutos de manzanos provenientes de una finca particular, implantado en el año 2001 en la localidad de San Luis del Palmar situada a $25 \mathrm{~km}$ al sudeste de Corrientes Capital (Longitud O: 58 $49^{\circ}$ Latitud S: $27^{\circ} 28^{\prime}$ Altura: $60 \mathrm{msm}$ ).

El lote consta de media hectárea con plantas de diferentes combinaciones de portainjertos y cultivares de manzanos conducidas en espaldera, en un marco de plantación de $2 \mathrm{~m}$ entre plantas y $4 \mathrm{~m}$ entre líneos, implantadas en un suelo franco arcilloso con características mólicas, con 1,7\% de materia orgánica, pH 6.2; 5 ppm de fósforo (Bray-Kurtz $\left.\mathrm{N}^{\circ} 1\right)$ y buenos contenidos de Calcio $\left(5.6 \mathrm{cmol} . \mathrm{kg}^{-1}\right)$ con una profundidad efectiva de suelo de aproximadamente $100 \mathrm{~cm}$.

Los cultivares estudiados pertenecen a los denominados de bajo requerimiento de horas de frío y son:

Caricia: resultado del cruzamiento de los cultivares Anna y Prima. Su exigencia de frío es de 350 horas, sus frutos tienen fondo crema verdoso con el $90 \%$ cubierto de rojo oscuro. La cosecha de este cultivar en Brasil es en el mes de enero.

Eva: resultado del cruzamiento de los cultivares Anna y Gala. Su exigencia de frío es de 300 a 350 horas, sus frutos más bien cónicos, con fondo color crema y amarillo, cubierto con el 30 a 70 por ciento de rojo vivo. La cosecha en Brasil es entre el 13 de diciembre y el 13 de enero.

Anna: resultado del cruzamiento de "Red Hadassiya” x "Golden Delicious" efectuado en Doar Na Shomron, Israel, por Abba Stein en 1959 (Brooks y Olmo, 1972). Su cultivo se ha extendido ampliamente por las zonas subtropicales del mundo por sus bajos requerimientos de horas de frío (150 a 200 horas). Sus frutos son redondo-cónicos a cónicos-alargados, con fondo amarillo y coloración rojo-rosada. En Brasil (Estados de São Paulo y Paraná) se cosecha en la segunda quincena de noviembre.

Estos cultivares están combinados con los portainjertos:

East Malling 9 (EM9): posee un sistema radi- cular de escasa penetración en el suelo, requiere alta fertilidad. Es el portainjerto más precoz para entrada en producción, pero poco tolerante al exceso de humedad. Por ser un pie semienanizante, ha permitido una máxima densidad de plantación con grandes producciones, aunque a costa de su longevidad.

Malling Merton 111 (MM111): posee un sistema radicular de buena penetración en el suelo, con una importante cabellera radicular, lo que le confiere cierta resistencia a la sequía. Es considerado un portainjerto semivigoroso, ofrece frutos de gran tamaño y calidad.

"Pie Correntino" (PC) pie criollo, probablemente proveniente de semilla. Actualmente es una planta de más de 100 años de edad, implantada en la localidad de San Luis del Palmar, con un muy buen desarrollo vegetativo y óptimo estado sanitario lo que ha motivado su elección para propagarla como portainjerto y el consecuente estudio de su comportamiento como tal.

En el lote se efectuaron las prácticas culturales necesarias para cubrir los requerimientos del cultivo. Las plantas se fertilizaron con un fertilizante complejo, $\mathrm{N} / \mathrm{P}_{2} \mathrm{O}_{5} / \mathrm{K}_{2} \mathrm{O} / \mathrm{MgO}$ en proporción 15:6:15:6, las dosis aplicadas fueron de 30; 40 y 50 g planta $^{-1}$ respectivamente en las tres campañas, dosificadas en 4 aplicaciones cada una: post cosecha (verano), otoño, prefloración y cuajado de frutos. Durante el período de maduración de los frutos, las plantas se suplementaron con nitrato de calcio $\left(\mathrm{CaNO}_{3}\right.$. N: 8.5\%; CaO: 16.3\%; MgO: 0.8\%) a razón de 30cc/10L. Conforme a un plan sanitario las plantas recibieron aplicaciones de fungicidas (oxicloruro de cobre $2,5 \%$, carbendazim $1 \%$ y estrobilurinas $0,2 \%$ ), y acaricidas e insecticidas (dicofol $2 \%$, dimetoato 1\%). En todas las campañas se realizó raleo manual de frutos siguiendo el criterio establecido por (Camilo y Pereira, 2002).

El diseño experimental es completamente al azar, parcela experimental una planta, con 4 repeticiones de las siguientes combinaciones: "Caricia”, "Eva" y "Anna” sobre PC; "Caricia” y "Anna” sobre EM9 y "Eva” y "Anna” sobre MM111. Los resultados se analizaron con Infostat (2002), realizándose ANOVA y Test de Tukey $(\mathrm{p} \leq 0,05)$ (Steel et al, 1992).

Se evaluó el comportamiento de las diferentes combinaciones desde el inicio de cada ciclo hasta caída de hojas o final del ciclo anual. Para ello se midieron en cuatro árboles de cada combinación, durante tres campañas seguidas, los siguientes parámetros:

a) Productividad: al momento de cosecha se contaron y pesaron los frutos de cada planta y combinación determinando cantidad de frutos y cosecha total por planta en estudio en $\mathrm{kg}$ árbol ${ }^{-1}$.

b) Diámetro de tronco: se estimó el vigor de las diferentes combinaciones por medio de medicio- 
nes de diámetro de tronco a $25 \mathrm{~cm}$ del suelo, al inicio (septiembre) y final (junio) del período vegetativo en cada ciclo.

c) Eficiencia del cultivo: con los datos de productividad y de área de tronco se calculó la eficiencia de cultivo (Lombard et al, 1988) de cada combinación.

\section{RESULTADOS Y DISCUSIÓN}

La productividad de las diferentes combinaciones durante las tres campañas, se presenta en el Gráfico 1. En este se observa que la cosecha promedio en kg. árbol ${ }^{-1}$ fue en aumento con el pasar de los años, siendo entre 1 a 4 kg. árbol ${ }^{-1}$ en la campaña 2003/2004 (plantas de 2 años); entre 4 a 7 kg. árbol ${ }^{-1}$ en la campaña 2004/2005 (plantas de 3 años) y de 7 a 9 kg. árbol ${ }^{-1}$ en el ciclo 2005/2006 (plantas de 4 años) dependiendo de cada combinación. Estos rendimientos son relativamente bajos ya que solo alcanzan el 57\% de los rendimientos obtenidos en Colonia Caroya (Córdoba) para "Eva” y “Caricia” en plantas de 2 años y el 50\% en plantas de 3 años (Páez Morón, 2005).

El incremento en kg. árbol ${ }^{-1}$ para todas las combinaciones en la campaña 2004/2005, respecto a la anterior, se debió principalmente a un aumento de la cantidad de frutos por árbol en detrimento del tamaño de los mismos. Como se observa en el gráfico 1-a correspondiente a la campaña 2003/2004, “Anna/PC” fue la combinación que dio frutos de mayor peso promedio (110 g) y la cantidad de frutos promedio por árbol que produjo este cultivar fue de 35 frutos. Sin embargo en la campaña 2004/2005 se confirma el hecho de que la cantidad de frutos árbol $^{-1}$ fue la que incidió sobre la disminución del tamaño de los mismos, ya que en ninguna de las combinaciones se cosecharon frutos de peso promedio superior a los $80 \mathrm{~g}_{\text {fruto }^{-1}}$, debido a la gran cantidad promedio de frutas árbol ${ }^{-1}$ (entre 60 y 100 frutos árbol ${ }^{-1}$ según combinación). Esto coincide con lo citado por (Camilo et al 2002) quienes dicen que en condiciones óptimas la mayoría de los cultivares de manzanas tienden a producir grandes cantidades de frutos. Característica no muy deseable debido al efecto negativo que esto ejerce sobre la calidad de los frutos y tamaño comercialmente deseado. En la campaña 2005/2006 (gráfico 1-c), la cantidad de frutos promedio por árbol en cada combinación, no disminuyó sustancialmente respecto a la campaña anterior, sin embargo el peso promedio de los frutos fue entre $79 \mathrm{~g}$ y $117 \mathrm{~g}$ dependiendo de la combinación, lo cual se tradujo en un aumento de la producción en kg. árbol ${ }^{-1}$ en comparación a las campañas anteriores.

Evaluando la productividad de las tres campañas es estudio (tabla 1a) no se observaron diferencias significativas entre combinaciones. Sin embargo de las mediciones realizadas en este estudio en com- binación con observaciones a campo, se destaca el comportamiento del cultivar Anna en todas las combinaciones, ya que siempre se comportó como una buena variedad productiva en kg. planta ${ }^{-1}$.

"Caricia” dio buenos rendimientos en el tercer ciclo con ambos portainjertos evaluados y fue el cultivar Eva el que mostró mayor variabilidad respecto a producción con ambos portainjertos en las tres campañas en estudio, y cuyo rendimiento promedio a lo largo de los tres ciclos dio las producciones más bajas con ambas combinaciones en estudio (Tabla 1-a).

Gráfico 1: Productividad de diferentes combinaciones portainjerto cultivar de manzanos de bajos requerimientos de horas de frío en San Luis del Palmar - Corrientes durante tres campañas a) campaña 2003/2004 b) campaña 2004/2005 c) campaña 2005/2006. Cantidad promedio de frutos por árbol; peso promedio de frutos (g); cosecha promedio por árbol (kg).
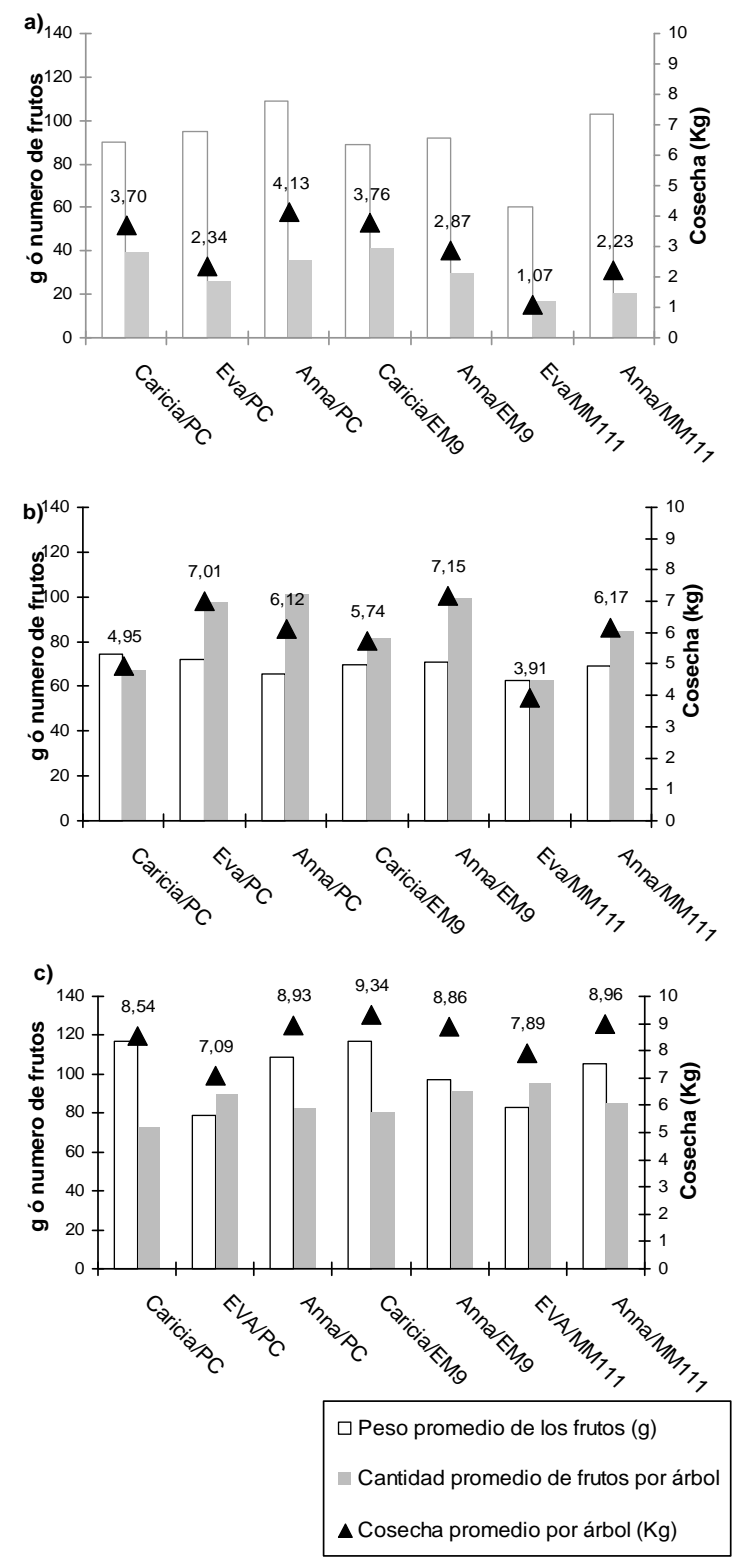
Tabla 1: Eficiencia de cultivo promedio de combinaciones de cultivares de manzanas de bajo requerimiento de horas de frío durante tres campañas. (a) Cosecha promedio en g por planta, (b) Área de tronco promedio en $\mathrm{cm}^{2}$ y (c) Eficiencia de cultivo promedio en $\mathrm{g}$. $\mathrm{cm}^{2-1}$ Test de Tukey, letras distintas indican diferencias significativas $(\mathrm{p} \leq 0,05)$.

\begin{tabular}{l|l|l}
\hline a) COSECHA (g/planta) & b) AREA DE TRONCO $\left(\mathrm{cm}^{2}\right)$ & c) EFICIENCIA $\left(\mathrm{g} / \mathrm{cm}^{2}\right)$ \\
\hline Eva/MM111 4289,92 a & Anna/EM9 23,67 a & Eva/MM111 117,83 a \\
Eva/PC 5480,83 a & Caricia/EM9 27,83 a & Eva/PC 142,00 ab \\
Caricia/PC 5729,75 a & Caricia/PC 29,08 a & Anna/MM111 186,83 ab \\
Anna/MM111 5783,17 a & Anna/MM111 31,25 a & Caricia/EM9 207,42 ab \\
Caricia/EM9 6280,17 a & Eva/MM111 33,08 a & Anna/PC 222,42 ab \\
Anna/EM9 6296,17 a & Anna/PC 34,58 a & Caricia/PC 231,50 ab \\
Anna/PC 6392,42 a & Eva/PC 39,30 a & Anna/EM9 279,00 b \\
\hline
\end{tabular}

Conforme al análisis de varianza $(\mathrm{p} \leq 0,05)$ no se encontraron diferencias significativas en lo que se refiere área de tronco de las diferentes combinaciones (Tabla 1-b). Las determinaciones por campaña se presentan en el gráfico 2. Como es de esperar el área de tronco de las diferentes combinaciones fue en aumento año a año. Si bien no se observaron diferencias significativas en relación a este parámetro, las combinaciones sobre EM9 siempre presentaron menos $\mathrm{cm}^{2}$ de tronco que los de las combinaciones sobre PC y MM111. Esto coincide con lo descripto para estos portainjertos, destacados por sus condiciones de semienanizante (EM9) y semivigoroso (MM111).

Del análisis de las tres campañas surge que las combinaciones sobre EM9 fueron las que presentaron menor área de tronco y las combinaciones sobre MM111 y sobre PC las de mayor área. Sin embargo las combinaciones sobre EM9 mostraron un comportamiento mas vigoroso en Corrientes (promedio tres campañas entre 23,67 y 27,83 cm²) en comparación con lo citado para este portainjerto por Denardi, (2002) en Santa Catarina Brasil $\left(16,2 \mathrm{~cm}^{2}\right)$. Lo mismo ocurre con MM111 el cual presentó un área de tronco promedio de entre 31,25 y 33,08 $\mathrm{cm}^{2}$ en Corrientes y lo citado para Brasil es de 22,6 $\mathrm{cm}^{2}$. En promedio el portainjerto "PC" en combinación con “Anna” y “Eva” presentó una mayor área de tronco que las combinaciones con MM111 (Tabla 1-b).

Las combinaciones sobre PC se caracterizaron por presentar a lo largo de las tres campañas el mismo comportamiento respecto al cultivar con el que se encontraban combinados. En combinación con Eva, PC se caracterizo por dar troncos con más diámetro en comparación a los otros dos cultivares. Esta característica podría deberse a que Eva fue un cultivar con baja productividad, favoreciendo así el crecimiento vegetativo de la combinación. Sin embargo el portainjerto $\mathrm{PC}$ en sí mismo, justifica más estudios para caracterizarlo detalladamente en aspectos sanitarios y adaptativos a nuestras condiciones edafoclimáticas.

Del promedio de las tres campañas, surge que la eficiencia de cultivo entre las combinaciones Eva/
Gráfico 2: Superficie de tronco $\left(\mathrm{cm}^{2}\right)$, y eficiencia de cultivo $\left(\mathrm{g} \mathrm{cm}^{2-1}\right)$ de combinaciones de cultivares de manzanas de bajo requerimiento de horas de frio sobre diferentes portainjerto durante tres campañas productivas. a) $2003 / 2004$; b) $2004 / 2005$ y c) $2005 / 2006$.
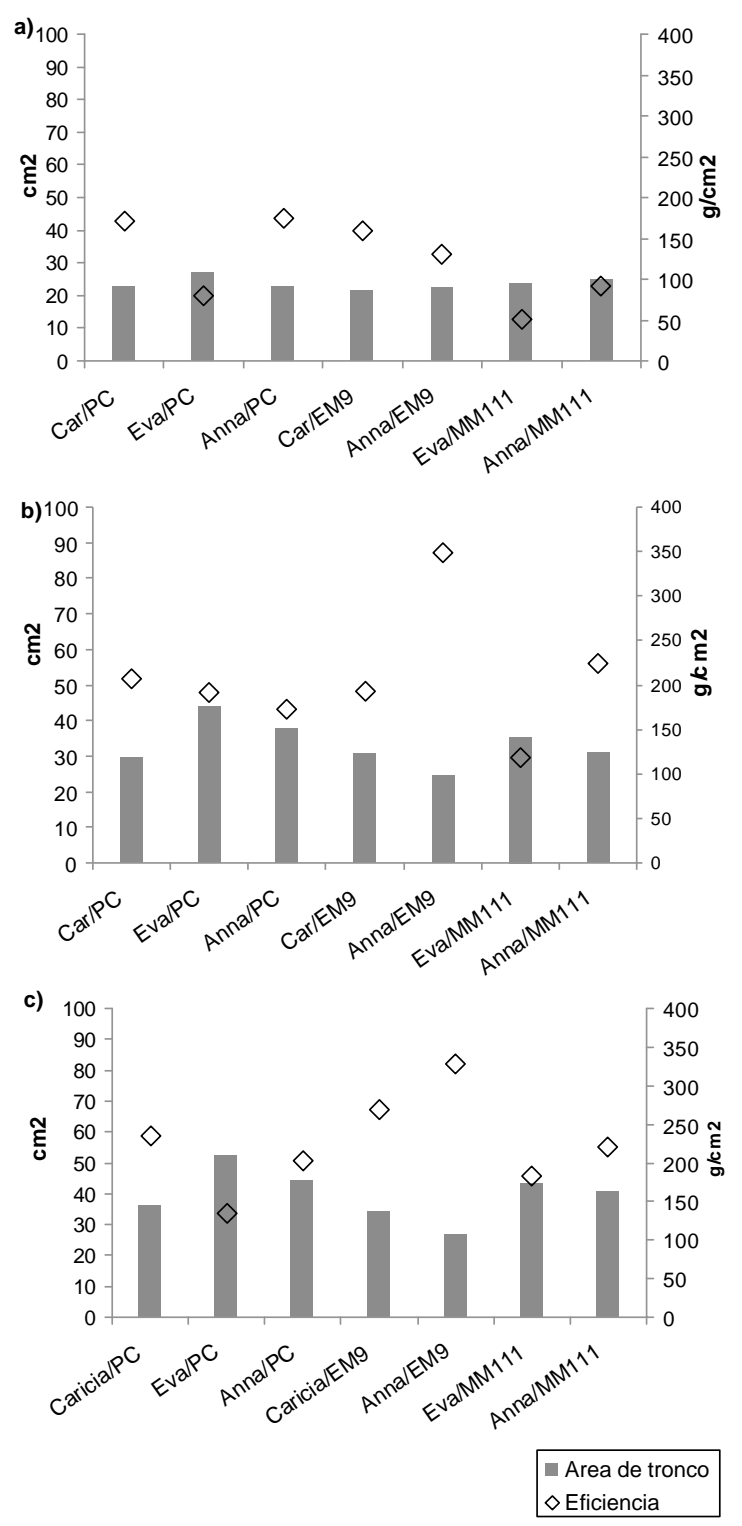
MM111 y Anna/EM9 presentó diferencias significativas conforme al análisis de varianza y Test de Tukey (Tabla 1-c).

En la combinación Eva/MM111 se observó una baja productividad en las dos primeras campañas en estudio y una productividad intermedia en la última, sin embargo debido al portainjerto semivigoroso (área de tronco) la eficiencia del cultivo resultó ser siempre baja (Gráfico 2). La combinación de Anna/EM9 dio en las tres campañas una buena cosecha comparada con las demás combinaciones e injertado sobre EM9, dio como resultado su alta eficiencia. Los rangos de eficiencia del cultivo citados por Lombard et al (1988) para cultivares de manzanos de zonas templadas se encuentra entre 300 y $1100 \mathrm{~g} \mathrm{~cm}^{-2}$ según cultivar. Es decir que en las campañas 2004/2005 y 2005/2006 (Gráfico 2 b y c) la eficiencia de cultivo de la combinación Anna/ EM9 se halló dentro de estos límites. Sin embargo para el resto de las variedades y combinaciones la eficiencia del cultivo está por debajo de lo citado, lo cual se explicaría por la combinación del alto vigor que presentan los portainjertos en estudio y la baja productividad en $\mathrm{kg}$ planta $^{-1}$ que dieron los diferentes cultivares en estas tres campañas.

\section{CONCLUSIONES}

De lo evaluado durante las campañas 2003/2004, 2004/2005 y 2005/2006 en manzanos de cultivares de bajos requerimientos de horas de frío implantados en Corrientes se concluye que su cultivo es factible en la zona.

Respecto a la eficiencia de cultivo se encontraron diferencias significativas entre las combinaciones Eva/MM111 y Anna/EM9, siendo el comportamiento de esta última combinación el más eficiente.

\section{BIBLIOGRAFÍA}

Brooks R.M. y Olmo H. P. (1972) Register of new fruit and nut varieties Berkeley: University of California 7-12

Camilo, A. N. y Pereira A. J. (2002) A cultura da macieira. Epagri. Rural de Santa Catarina S.A. Florianópolis Brasil. Capítulo 14: 419 -461.

Denardi, F. (2002) A cultura da macieira. Epagri. Rural de Santa Catarina S.A. Florianópolis Brasil. Capítulo 6: 169-227

INFOSTAT (2002) Infostat versión 1.1. Manual de usuario. Grupo Infostat. FCA, Universidad Nacional de Córdoba. Primera edición, Editorial Brujas, Argentina.

Lombard, P.B., Callan, N.W., Dennis, F.G. (Jr.), Looney, N.E., Martin, G.C., Renquist, A.R., \& Mielke, E.A. (1988). Towards a Standardized Nomenclature, Procedures, Values, and Units in Determining Fruit and Nut Tree Yield Performance. Hort Science 23(5): 813-817.

Páez Moron, P.G. (2005) Guía para producción de manzanas de bajos requerimientos de horas de frío en Colonia Caroya - Córdoba Ediciones INTA Boletín No 11.

Provasi, A.A.; Pieckun, A. A.; Rybak, M.; Lasserre, S. R.; Bogado, E.F. (2000) Posibilidad de producción y comercialización de manzanas subtropicales en Misiones, Miscelánea $N^{\circ} 43$ p.10 EEA INTA Cerro Azul.

Samson, J.A. (1991) Fruticultura tropical. Editorial Limusa. DF México. 395 páginas.

Steel, R. y Torrie J. (1992) Bioestadística: Principios y Procedimientos. Ed. Mc Graw Hill. México 622pp. 